\title{
Effects of Apigenin Treatment on Random Skin Flap Survival in Rats
}

\author{
Xinyi $\mathrm{Ma}^{1,2 t}$, Yuting $\mathrm{Lin}^{3 \dagger}$, yingying $\mathrm{Liu}^{2}$, Wenjie $\mathrm{Li}^{2}$, Jibing $\mathrm{He}^{2}$, Miaojie Fang ${ }^{2}$ and \\ Dingsheng Lin $^{1 *}$
}

\begin{abstract}
${ }^{1}$ Department of Hand and Plastic Surgery, The Second Affiliated Hospital and Yuying Children's Hospital of Wenzhou Medical University, Wenzhou, China, ${ }^{2}$ Second College of Clinical Medical, Wenzhou Medical University, Wenzhou, China, ${ }^{3}$ First College of Clinical Medical, Wenzhou Medical University, Wenzhou, China
\end{abstract}

Random skin flaps are often used in plastic surgery, but the complications of marginal flap ischemia and necrosis often limit their wider clinical application. Apigenin (Api) is a flavonoid found in various fruits and vegetables. Api has been shown to promote angiogenesis, as well as reduce oxidative stress, membrane damage, and inflammation. In this study, we assessed the effects of Api treatment on random skin flap survival. Dorsal McFarlane skin flaps were transplanted into rats, which were randomly divided into three groups: control (normal saline), low-dose Api (20 mg/kg), and high-dose Api (50 mg/kg). Seven days after the surgery, the activity of superoxide dismutase (SOD) and the level of malondialdehyde

OPEN ACCESS

Edited by:

Peter Vee Sin Lee,

The University of Melbourne, Australia

Reviewed by:

Yun Qian,

Shanghai Jiao Tong University, China

Fabio Vivarelli,

University of Bologna, Italy

*Correspondence:

Dingsheng Lin

lindingsheng@gmail.com

${ }^{\dagger}$ These authors have contributed equally to this work

Specialty section:

This article was submitted to Translational Pharmacology, a section of the journal Frontiers in Pharmacology

Received: 04 November 2020 Accepted: 04 January 2021

Published: 24 February 2021

Citation:

Ma X, Lin Y, Liu Y, Li W, He J, Fang $M$ and Lin D (2021) Effects of Apigenin

Treatment on Random Skin Flap

Survival in Rats.

Front. Pharmacol. 12:625733.

doi: 10.3389/fphar.2021.625733
(MDA) were measured. Histological analyses were performed to determine flap survival and tissue edema. H\&E staining was performed to assess the histopathological changes in skin flaps, and the levels of microvascular density (MVD) were determined. Laser doppler flowmetry was used to assess microcirculation blood flow. Flap angiography was performed by injection of lead oxide/gelatin. The expression levels of vascular endothelial growth factor (VEGF), tumor necrosis factor- $\alpha$ (TNF- $\alpha$ ), interleukin-6 (IL-6), and interlukin-1 $\beta$ (IL-I $)$ were evaluated by immunohistochemistry. Rats in the high-dose Api group exhibited higher average flap survival area, microcirculatory flow, increased SOD activity, and higher VEGF expression levels compared with the other two groups. Furthermore, the levels of MDA and pro-inflammatory cytokines were significantly decreased in rats treated with high-dose Api. Our findings suggest the potential usefulness of Api in preventing skin flap tissue necrosis.

Keywords: apigenin, random skin flap, inflammation, oxidative stress, microangiogenesis

\section{INTRODUCTION}

In contrast to axial pattern flaps, random skin flaps lack axial vascularization, due to anatomical differences in blood supply. Flap design and direction are not affected by the distribution of axial blood vessels; therefore, flaps are designed in a way to minimize tissue or organ dislocation during plastic surgery and cosmetic defect repairment. Since random skin flaps lack specific blood vessels, flap survival relies on blood supply from the pedicle muscle skin. Hence, low perfusion pressure in these tissues impacts flap survival, and marginal flap ischemia and necrosis, observed in 10-15\% of cases (Dolan et al., 1995; Lille et al., 1999), limit their wide clinical application.

The development of interventions to prevent or treat skin flap necrosis remains an unmet clinical need. The length-to-width ratio of the flap should not exceed 2:1 because the lack of flap vascularization increases the risk of distal ischemia and necrosis. In the face and neck, the 
length-to-width ratio can be increased to $2.5: 1$, due to the good vascularization of these tissues. In random skin flaps, the scarfskin of the terminal necrotic area is characterized by marked inflammatory cell infiltration and coagulation necrosis (Ekin et al., 2019). Furthermore, inflammatory responses and ischemia reperfusion injury contribute to random skin flap necrosis. Ischemic flap tissues are characterized by extensive cell membrane damage and increased levels of arachidonic acid metabolites, promoting the recruitment of neutrophils and other inflammatory cells. The production of reactive oxygen species (ROS) and other inflammatory mediators by these immune cells aggravate ischemia and tissue damage (Kang et al., 2014). Therefore, the development of strategies to promote angiogenesis and improve local blood supply, as well as inhibit the production of inflammatory mediators and ROS, is crucial for improving the survival of random skin flaps.

Apigenin (Api) or $4^{\prime}, 5,7$-trihydroxyflavone is a naturally occurring flavonoid, abundant in various vegetables, fruits, beans, and tea leaves, among which celery has the highest content. Api contains $4^{\prime}$ hydroxyl groups at positions 5 and 7, as well as a $\mathrm{C} 2 \mathrm{C} 3$ double bond largely responsible for its unique physicochemical properties (Miean and Mohamed, 2001; Skerget et al., 2005). Although Api is not water-soluble, it can be dissolved in ethanol, dimethyl sulfoxide (DMSO), and low-concentration potassium hydroxide solution. Pure Api has a light yellow or yellow-green color. The phenol ring of Api can bind to different sugar groups, including glucose and glycoside ligands. Celery, an Api-rich plant, contains high levels of antioxidants, which have been shown to reduce oxidative stress and protect from numerous human diseases, including cancer (Nabavi et al., 2015), coronary heart disease ( $\mathrm{Hu}$ et al., 2015), and aging. Api exerts antiinflammatory effects by inhibiting immune cell production of tumor necrosis factor- $\alpha$ (TNF- $\alpha$ ), interlukin-6 (IL-6), and interlukin-1 $\beta$ (IL-1 $\beta$ ) production. Api promotes vascular regeneration after ischemia by enhancing the expression of vascular endothelial growth factor (VEGF) (Miean and Mohamed, 2001; Skerget et al., 2005). As a natural antioxidant, Api is also believed to lower blood pressure, prevent atherosclerosis, and suppress tumor growth (Telange et al., 2017). Compared with other flavonoids, Api has low toxicity and mutagenicity (Xu P. F. et al., 2017).

Due to its anti-inflammatory, antioxidant, and pro-angiogenic effects, Api treatment has been considered as a method to improve random skin flap survival (Bachle et al., 2011; Krag et al., 2017; Pang et al., 2018; Ren et al., 2018). In this study, we established a rat model of random skin flap, and assessed the effects of Api on flap survival.

\section{MATERIALS AND METHODS}

\section{Animals and Reagents}

Ethical approval for this study was provided by the Laboratory Animal Ethics Committee of Wenzhou Medical University, Wenzhou, China (Chairperson, Prof. Shengwei Jin) on February 28, 2018 (no. WYDW2017-0509). Pathogen-free Sprague-Dawley male rats (200-250 g, 2-3 months old) were obtained from the Wenzhou Medical University Laboratory Animal Center. Api (purity $298 \%$ ) was purchased from Solarbio life sciences (Beijing, China). Api was dissolved in $4 \%$ $(\mathrm{v} / \mathrm{v}) \mathrm{DMSO}$, and corn oil was added to achieve a concentration of $4 \mathrm{mg} / \mathrm{ml}$.

\section{Establishment of a Rat Flap Model}

Rats $(n=60)$ were divided into three groups: high-dose (50 mg/kg) Api, low-dose (20 mg/kg) Api, and control ( $\mathrm{n}=20$ animals per group). Rats were not fed the night before the experiment. The next day, rats were anesthetized by intraperitoneal injection of pentobarbital sodium saline solution $(40 \mathrm{mg} / \mathrm{kg})$. After back hair removal, the modified McFarlane $(3 \times 9 \mathrm{~cm})$ flap was applied to the middle back of each rat (Figure 1A). The pedicle artery perforator flaps were sutured with 4-0 nylon sutures. To prevent shock, we intraperitoneally injected $50 \mathrm{ml} / \mathrm{kg}$ of normal saline. For postoperative observation, the flaps were divided into three types: proximal area (zone 1), middle area (zone 2), and distal area (zone 3$)$. After surgery, rats were single-caged $\left(25^{\circ} \mathrm{C}, 40-60 \%\right.$ humidity) and given ad libitum access to water and food. Rats in the high-dose Api and low-dose Api groups were intragastrically administered 20 and $50 \mathrm{mg} / \mathrm{kg}$ Api, respectively, per day; rats in the control group were administered corn oil and DMSO. The operation was performed daily at $3 \mathrm{pm}$ for seven consecutive days. All procedures were performed by the same person to minimize experimental errors.

\section{Macroscopic Flap Survival Assessment}

Flap survival was macroscopically examined at days $1,3,5$, and 7; changes in color, texture, tissue elasticity, hair growth, and tissue necrosis were recorded. Skin flap necrosis was defined as blackened color, stiff texture, shrunken tissue, reduced elasticity, and absence of bleeding after tissue puncture. The flap survival area was assessed 7 days after the operation by measuring the surviving area and total area of each flap using weighing paper and the weights of the flap using an electronic scale. Flap survival quantification was achieved using the following formula: flap survival area $(\%)=$ paperweight of the flap survival area/paperweight of the total flap surface area $\times 100$.

\section{Histopathological Examination}

Rats were sacrificed 7 days after the operation by anesthetic overdose. Tissue samples $(1 \times 1 \mathrm{~cm})$ were obtained from the proximal, middle, and terminal skin flaps (zones 1, 2, and 3, respectively), fixed in $4 \%$ paraformaldehyde for $24 \mathrm{~h}$, and paraffin-embedded. Tissue sections were prepared, followed by dewaxing, dehydration, staining, and microscopic examination. The degrees of tissue edema, necrosis, and neutrophil infiltration were evaluated under a light microscope (magnification, $\times 100$ ). Microvascular density (MVD) was used to assess angiogenic aggregation. Five optical fields were randomly selected for each tissue section, and the microvessel number in the crosssection was counted; the average number of microvessels per square millimeter area was calculated and the microvessel density per unit area was used to evaluate MVD. 

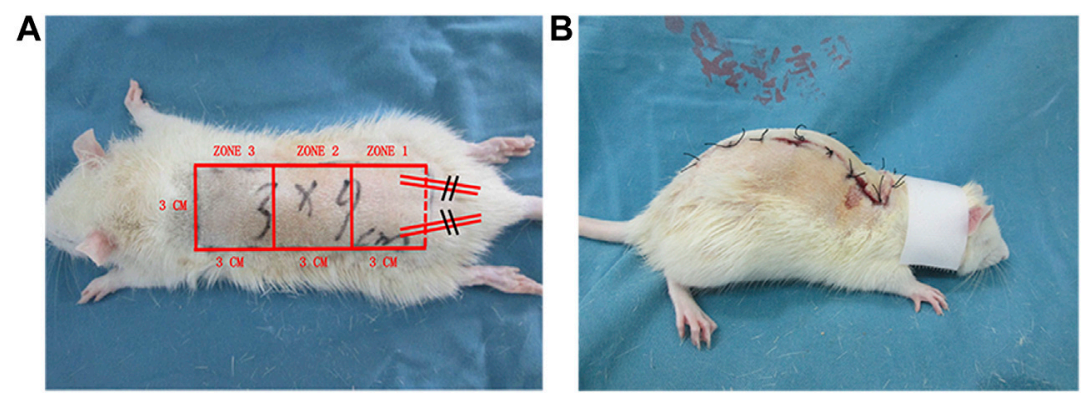

FIGURE 1 | (A) A modified McFarlane flap was established on the dorsum of each rat. (B) A patent neck cover was used to prevent the rat from chewing the flap.

\section{Laser Doppler Flowmetry}

Seven days after the operation, rats were anesthetized and restrained. The blood flow in each area of the flap (proximal area, middle area, and distal area) was measured using a laser Doppler imaging system. Microcirculatory blood flow in the local tissues was expressed as perfusion units (PU).

\section{Gelatin/Lead Oxide Angiography}

Rats were sacrificed 7 days after the operation by anesthetic overdose. Pre-warmed $\left(37^{\circ} \mathrm{C}\right)$ isotonic saline was injected into the carotid artery to clean blood vessels, and the blood was drained through the jugular vein; body temperature was monitored throughout this process. Subsequently, gelatin/lead oxide solution $(100 \mathrm{ml} / \mathrm{kg})$ was injected into the carotid artery; perfusion was continued until the solution reached the limbs, ears, and corneas. The dorsal flap and cutis were dissected and subjected to X-ray angiography.

\section{Immunohistochemical Staining}

Paraffin-embedded sections were stained using the Elivison twostep method. Sections were incubated with normal goat serum blocking solution and left to stand at room temperature $\left(20-25^{\circ} \mathrm{C}\right)$ for $25 \mathrm{~min}$. Tissue sections were then incubated with a mouse anti-rat VEGF primary antibody (1:100 in $50 \mu \mathrm{l})$ overnight at $4^{\circ} \mathrm{C}$. Subsequently, sections were incubated at $37^{\circ} \mathrm{C}$ for $45 \mathrm{~min}$, washed with phosphate-buffered saline (PBS), and incubated with a goat anti-mouse secondary antibody (1:50 in $50 \mu \mathrm{l})$ for $1 \mathrm{~h}$ at $37^{\circ} \mathrm{C}$. After washing with PBS, tissue sections were incubated with diaminobenzidine for $10 \mathrm{~min}$. Sections were observed under low-power and high-power light microscopes. Five optical fields per section were imaged and used for quantification of VEGF expression. The basic principle of field selection in the immunohistochemical analysis was to randomly select five fields that did not overlap under a $400 \times$ light microscope. Similar methods were used to detect the expression level of TNF- $\alpha$, IL- 6 and IL- $1 \beta$.

\section{TNF- $\alpha$ and IL- 6 Serum Levels}

Blood was centrifuged at $5,000 \times \mathrm{g}$ for $15 \mathrm{~min}$, serum was collected, and serum TNF- $\alpha$ and IL- 6 levels were measured using an ELISA kit according to the manufacturer's instructions. Optical absorbance was measured at $450 \mathrm{~nm}$.

\section{Superoxide Dismutase (SOD) Activity and Malondialdehyde (MDA) Content Measurements}

Ischemia reperfusion injury typically occurs within $24-48 \mathrm{~h}$ of flap transplantation. The rats were sacrificed by anesthetic overdose 7 days after the surgery. Tissue samples $(0.5 \times$ $0.5 \mathrm{~cm}$ ) were collected from the middle area (zone 2), where partial flap necrosis was observed. Samples were homogenized, weighed, and diluted in an ice bath to $10 \%$ of their initial concentration. The MDA level and SOD activity were measured according to the manufacturer's instructions.

\section{Statistical Analysis}

Statistical analyses were performed using SPSS version 19 (Chicago, IL, United States). Data were expressed as mean \pm standard error of the mean (SEM). The mean values of different groups were compared using the Student's $t$-test. $p$-values $<0.05$ were considered statistically significant.

\section{RESULTS}

\section{Effects of Api on Flap Survival and Features}

On postoperative day 1, all animals presented some degree of swelling near the incision edges of zone 3 , accompanied by dark brown patches. However, there was no profound tissue swelling in zones 1 and 2. Seven days after the operation, we observed a demarcation line between the healthy area of the flap and the necrotic tissue. The necrotic tissue, especially in the distal flap, was dark and hard, with reduced tissue elasticity and granulation. Macroscopic examination revealed that the extent of necrosis was greater in the control group compared to that in Api-treated rats (Figure 2A). The flap survival rate was markedly reduced in the control group $(49.18 \pm 2.83 \%)$ compared to that in the low-dose Api and high-dose Api groups (70.66 \pm 2.18 and $84.51 \pm 3.85 \%$, respectively; Figure 2B). The degree of tissue edema was evaluated by measuring the tissue water content. The percent tissue water in the high-dose Api, low-dose Api, and control groups were $25.86 \pm 3.49,38.23 \pm 3.61$, and $60.88 \% \pm 6.59 \%$, respectively (Figure $2 \mathrm{C}$ ). The differences in the extent of tissue edema among the groups were statistically significant $(p<0.01)$. 

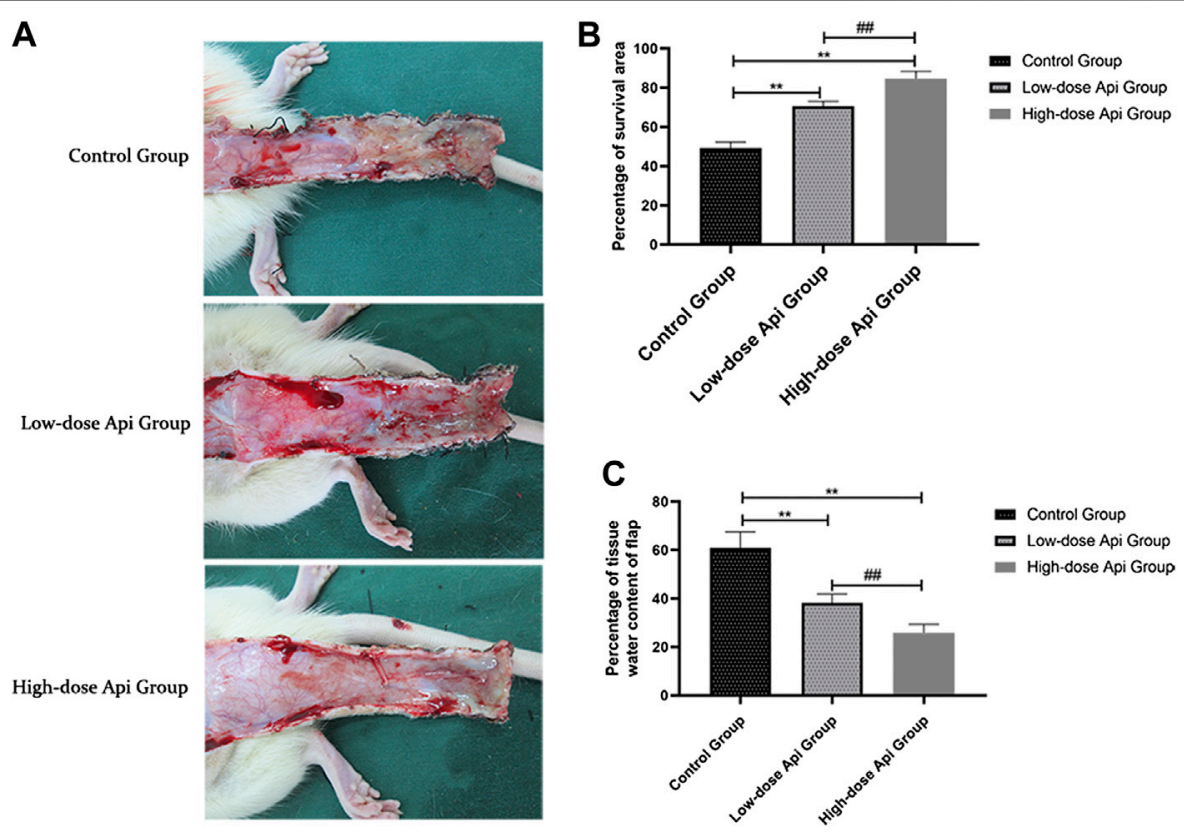

FIGURE 2 | (A) Digital photographs of dorsal skin flaps in rats from the three groups. Macroscopic comparison of the overall appearance of the surviving and necrotic areas of the flap. (B) Percentage of survival area on day 7. Api increased the survival rate of skin flaps. (C) Percentage of tissue water content on day 7. Api reduced the degree of tissue edema in skin flaps. ${ }^{*} p<0.01$ vs. control group. ${ }^{\# \#} p<0.01$ vs. Low-dose Api group.

A

Low-dose Api Group

High-dose Api Group
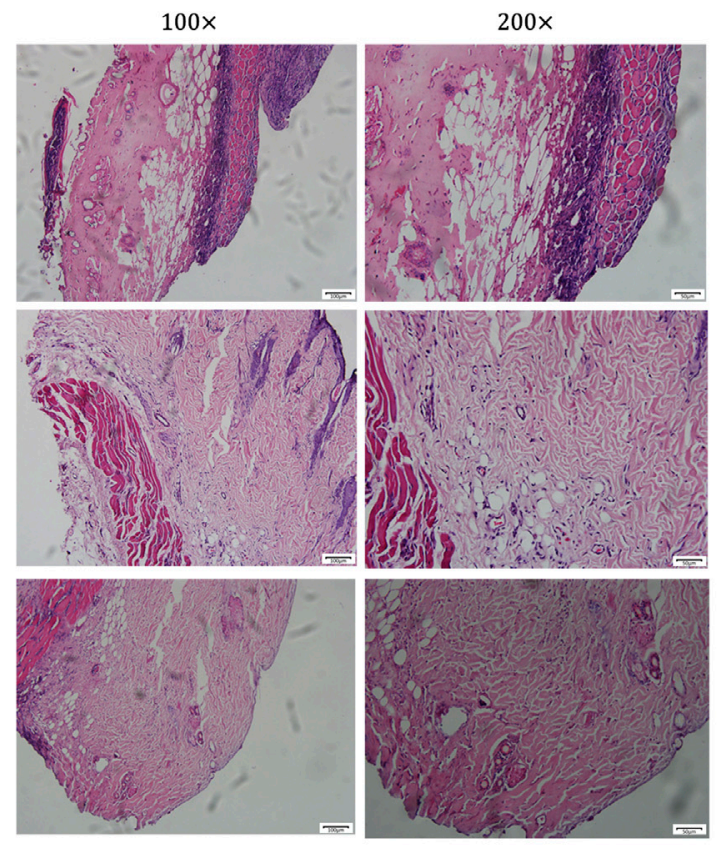

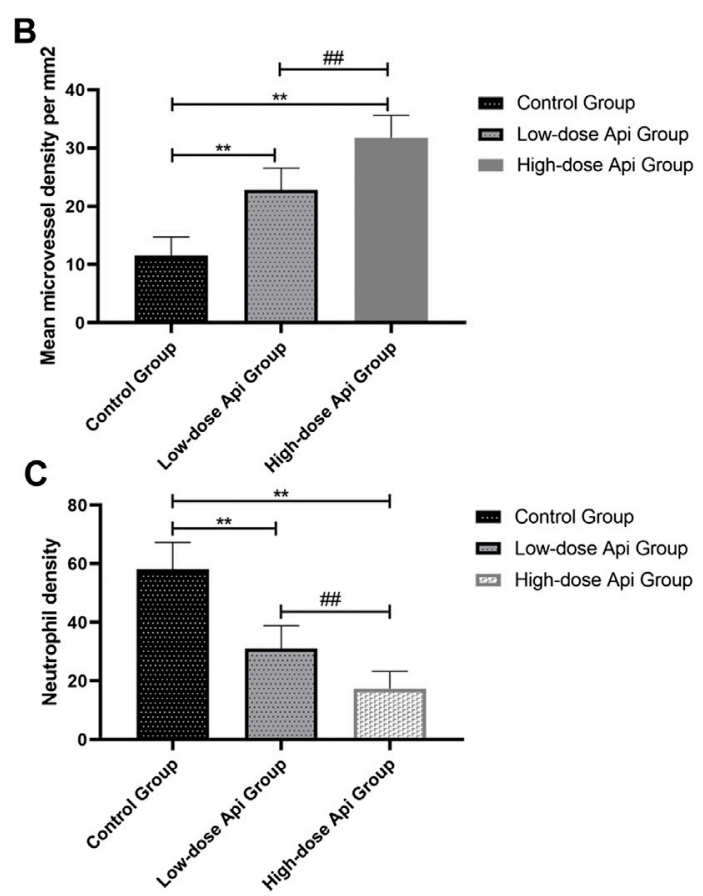

FIGURE 3 | (A) Histopathological features of the flaps were assessed using hematoxylin and eosin (H\&E) staining on day 7 after the operation; representative images acquired with a light microscope are shown (magnification, $\times 100, \times 200$ ). Api reduced histopathological damage. (B) The microvascular density in zone 2 of the dorsal flap on day 7 after the operation. Api increased microvessel density. (C) Neutrophil density on day 7 after the operation. Api reduced neutrophil density. ${ }^{* *} p<0.01$ vs. control group. ${ }^{\# \#} p<0.01$ vs. Low-dose Api group. 

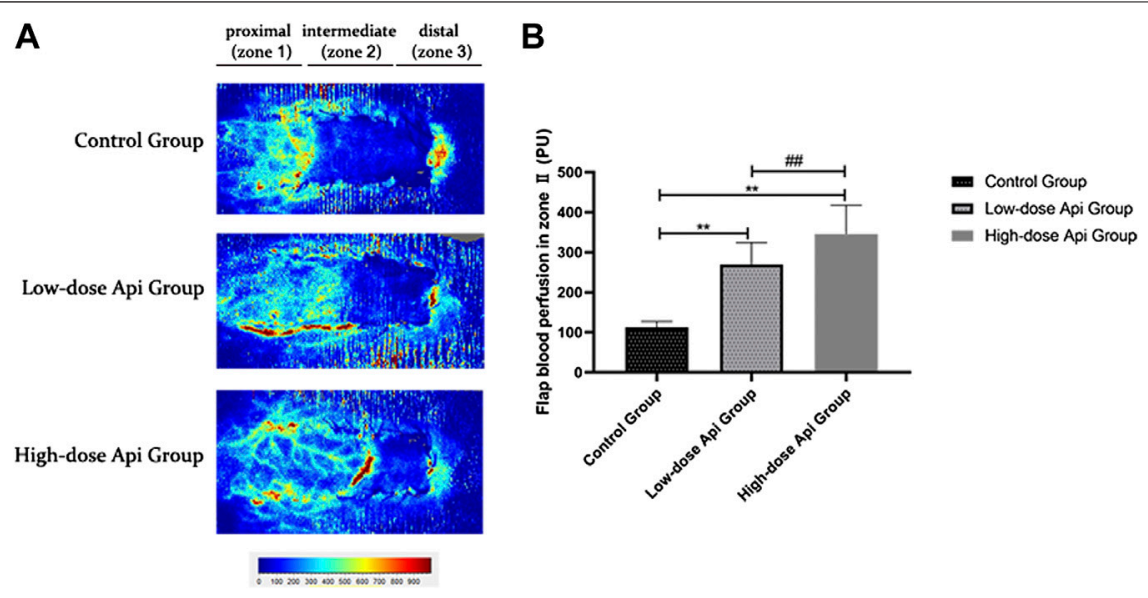

FIGURE 4 | (A) Laser Doppler flowmetry angiography showing blood perfusion on day 7 in the different groups. (B) Quantification of blood perfusion in zone 2 of the flap. Api increased blood flow. ${ }^{\star \star} p<0.01$ vs. control group. ${ }^{\# \#} p<0.01$ vs. Low-dose Api group.

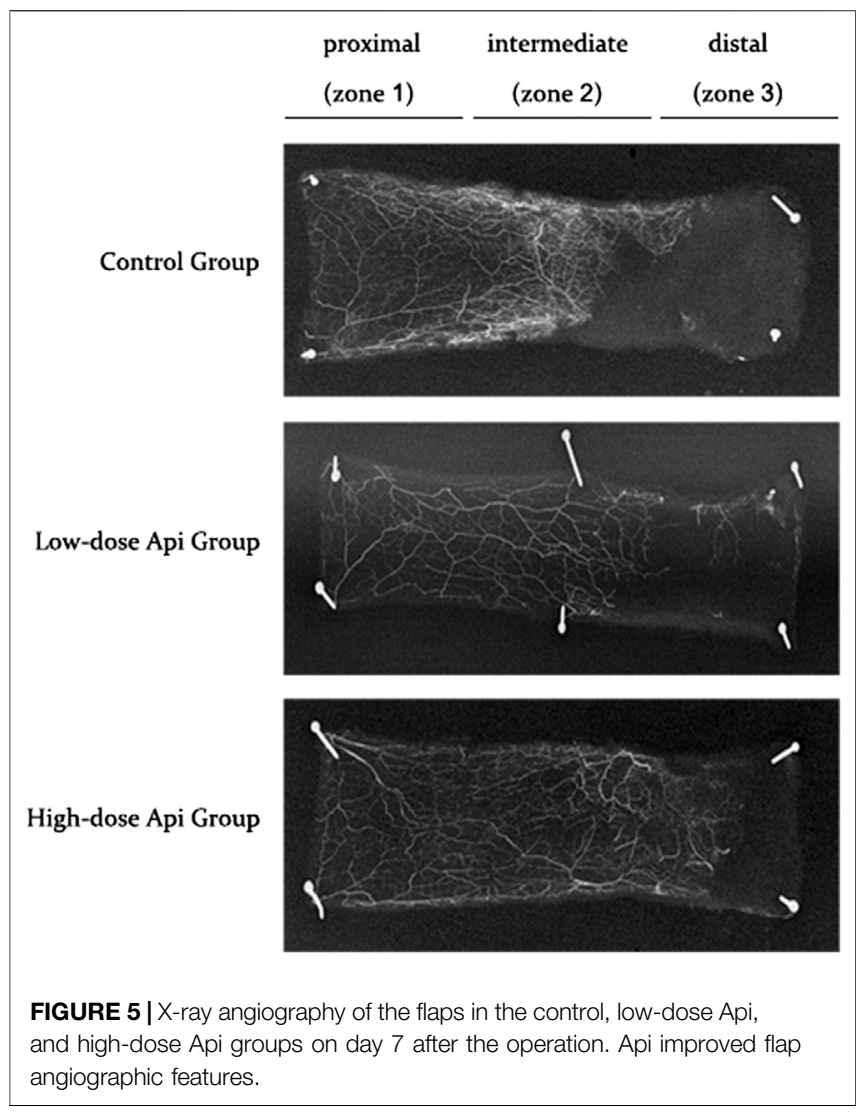

\section{Api Prevents Histopathological Skin Flap Damage}

In contrast with the flaps of Api-treated animals, the flaps of the control rats presented profound structural and histological abnormalities, including excessive inflammatory cell infiltration, reduced neovascularization, reduced fibroblast proliferation, granulation tissue thinning, and tissue edema
(Figure 3A). Furthermore, the MVDs of zone II in the highdose Api group $\left(31.75 \pm 3.85 / \mathrm{mm}^{2}\right)$ and low-dose Api group $\left(22.81 \pm 3.75 / \mathrm{mm}^{2}\right)$ were significantly higher than that in the control group (11.58 $\pm 3.12 / \mathrm{mm}^{2} ; p<0.01$; Figure 3B). Moreover, the neutrophil density in the low-dose Api group $\left(31.05 \pm 7.79 / \mathrm{mm}^{2}\right)$ and high-dose Api group $\left(17.37 \pm 5.83 / \mathrm{mm}^{2}\right)$ was significantly lower than that in the control group (58.07 \pm $9.26 / \mathrm{mm}^{2} ; p<0.01$; Figure 3C).

\section{Api Improves Blood Flow in Zone 2}

LDF angiography revealed that flap blood perfusion in zone 2 was significantly higher in the low-dose Api group (269.72 $\pm 54.58 \mathrm{PU})$ and high-dose Api group ( $345.65 \pm 71.87 \mathrm{PU})$ compared with that in the control group (112.53 $\pm 14.88 \mathrm{PU} ; p<0.01$; Figure 4).

\section{Api Promotes Skin Flap Neovascularization} Gelatine/lead oxide angiography 7 days after the operation indicated that neovascularization in the low-dose and high-dose Api groups was significantly higher than in the control group (Figure 5).

\section{Effects of Api on VEGF, IL-6, IL-1 $\beta$, and TNF- $\alpha$ Levels}

Immunohistochemical observations revealed that Api enhanced VEGF expression and downregulated the expression of proinflammatory cytokines in a dose-dependent manner. The levels of VEGF in the high-dose Api, low-dose Api, and control groups were 4,737.67 $\pm 267.01 \mathrm{IA}, 3,090.00 \pm 493.32 \mathrm{IA}$, and $1,665.83 \pm$ 248.91 IA, respectively $(p<0.01)$. The levels of IL-6 in the highdose Api, low-dose Api, and control groups were $819.83 \pm 115.31 \mathrm{IA}$, $1,996.33 \pm 230.22 \mathrm{IA}$, and 3,710.17 $\pm 225.38 \mathrm{IA}$, respectively $(p<$ $0.01)$. Similarly, IL-1 $\beta$ levels in the high-dose Api, low-dose Api, and control groups were $721.67 \pm 105.91 \mathrm{IA}, 1,293.83 \pm 239.67 \mathrm{IA}$, and $2,253.33 \pm 373.67 \mathrm{IA}$, respectively $(p<0.01)$. The levels of TNF- $\alpha$ in the high-dose Api, low-dose Api, and control groups were 1,196.33 \pm $159.29 \mathrm{IA}, 2,402.5 \pm 271.01 \mathrm{IA}$, and $4,223.00 \pm 206.92 \mathrm{IA}$, respectively $(p<0.01$; Figure 6$)$. 

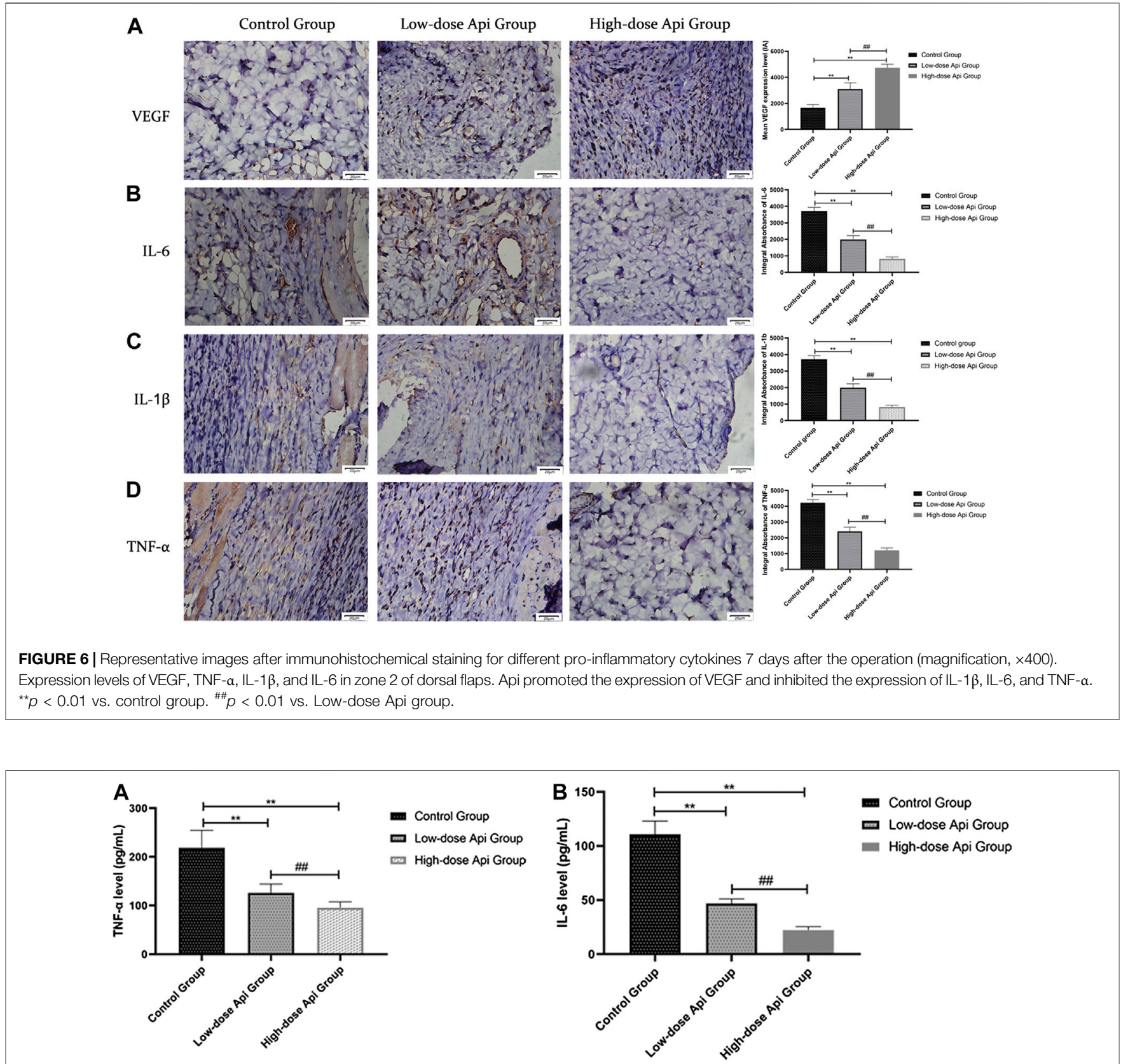

FIGURE 7|Serum levels of TNF- $\alpha$ (A) and IL-6 (B) 7 days after the operation as determined by ELISA. Api inhibited the expression of TNF- $\alpha$ and IL-6. ${ }^{\star \star} p<0.01$ vs. control group. ${ }^{\# \#} p<0.01$ vs. Low-dose Api group.

\section{Effects of Api on Pro-Inflammatory Cytokine Production}

The serum levels of pro-inflammatory cytokines were determined by ELISA. We found that the levels of TNF- $\alpha$ were significantly lower in the high-dose Api group $(95.62 \pm 12.11 \mathrm{pg} / \mathrm{ml})$ and lowdose Api group (125.83 $\pm 18.65 \mathrm{pg} / \mathrm{ml})$ compared with those in the control group $(218.36 \pm 36.21 \mathrm{pg} / \mathrm{ml} ; p<0.01$; Figure 7A). Furthermore, serum IL-6 levels were profoundly lower in the high-dose Api group $(22.25 \pm 3.11 \mathrm{pg} / \mathrm{ml})$ and low-dose Api group $(46.89 \pm 4.12 \mathrm{pg} / \mathrm{ml})$ compared with those in the control group (110.93 $\pm 12.08 \mathrm{pg} / \mathrm{ml} ; p<0.01$; Figure $7 \mathbf{B})$.

\section{Effects of Api on SOD Activity and the MDA Level}

Api treatment enhanced SOD activity and reduced the MDA level in a dose-dependent manner. Particularly, SOD activity in the low-dose Api group (51.12 \pm 6.36 units-mg-1-protein-1) and high-dose Api 

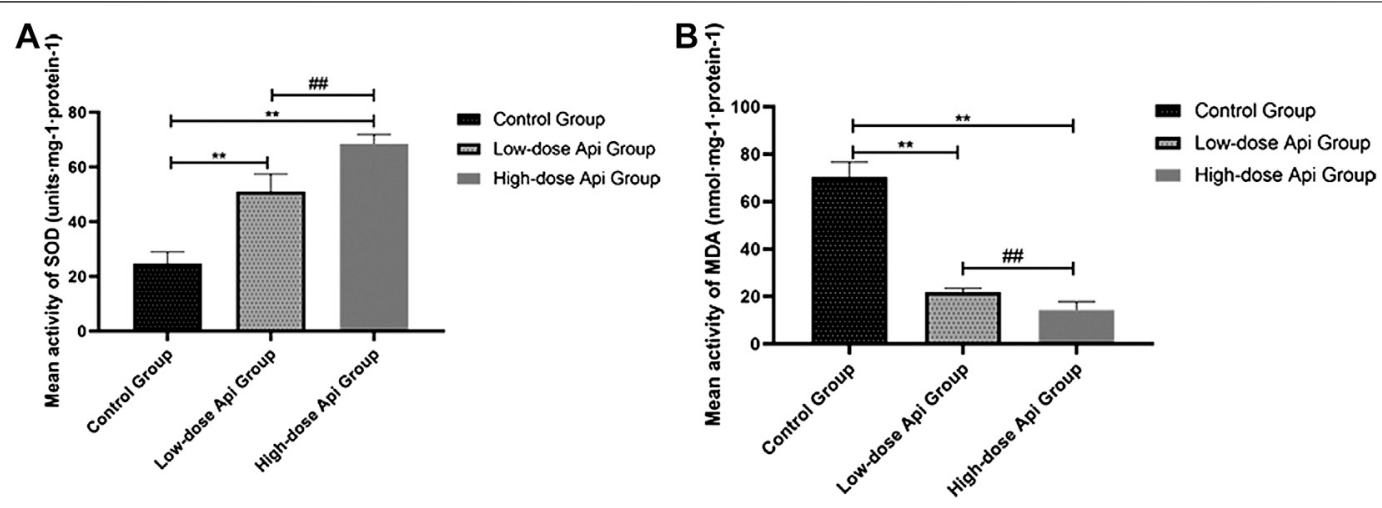

FIGURE $8 \mid$ (A) Mean SOD activity on day 7. (B) Mean MDA content on day 7. Api enhanced SOD activity and inhibited the expression of MDA. ${ }^{* \star} p<0.01$ vs. control group. ${ }^{\# \#} p<0.01$ vs. Low-dose Api group.

group $(68.52 \pm 3.42$ units.mg-1.protein-1) were significantly higher compared with that in the control group $(24.81 \pm 4.23$ units.mg1 .protein-1; $p<0.01$; Figure 8A). Conversely, MDA levels in the lowdose Api group (21.89 \pm 1.60 units-mg-1·protein-1) and high-dose Api group (14.29 \pm 3.53 units.mg-1.protein-1) were significantly lower compared with those in the control group $(70.49 \pm 6.25$ units.mg-1.protein-1; $p<0.01$; Figure 8B).

\section{DISCUSSION}

Api is a flavonoid found at higher levels in celery. Several lines of evidence have shown that it promotes tissue healing and angiogenesis, and prevents venous thrombosis. Additionally, Api can reduce oxidative stress and inflammation and has an excellent safety profile in humans (Ren et al., 2018; Salehi et al., 2019; Zhou et al., 2019). A previous study showed that Api (20 mg/kg/day) administration significantly attenuated early brain injury, which includes brain edema, blood-brain barrier disruption, neurological deficiency, and cell apoptosis, after subarachnoid hemorrhage in rats by suppressing the expression of toll-like receptor 4 (TLR4), nuclear factor- $\kappa \mathrm{B}(\mathrm{NF}-\mathrm{kB})$, and their downstream pro-inflammatory cytokines in the cortex and by upregulating the expression of tight junction proteins of the blood-brain barrier (Zhang et al., 2015). In addition, a diabetes study showed that Api ( $20 \mathrm{mg} / \mathrm{kg} /$ day) improves renal dysfunction and oxidative stress (Malik et al., 2017). It also prevented mitogen-activated protein kinase activation, which inhibited inflammation (reduced TNF- $\alpha$, IL-6, and NF- $\kappa B$ expression) and apoptosis (increased expression of $\mathrm{Bcl}-2$ and decreased expression of Bax and caspase-3). Api (50 mg/kg/day) significantly improved paraquat-induced acute lung injury in mice (Luan et al., 2016). These findings indicate that Api administration decreases biochemical parameters of inflammation and oxidative stress, and improves oxygenation and lung edema, in a dosedependent manner. All of these effects are likely to improve the survival of ischemic flaps; thus, we chose 20 and $50 \mathrm{mg} / \mathrm{kg} /$ day as therapeutic Api doses.

In this study, we showed that intragastrical administration of Api in rats induced the expression of VEGF, promoting angiogenesis.
Additionally, it reduced oxidative stress and tissue inflammation, as well as inhibited ischemia reperfusion injury in skin flaps, reducing tissue edema, and improving flap survival. Our team has been exploring methods to intervene in the necrosis of ischemic flaps and has found that azadirachtin A (He et al., 2020), dexmedetomidine (Fang et al., 2020), and nobiletin (Jiang et al., 2020) have positive effects on flap survival. However, Api has better anti-inflammatory effects, reducing the release of inflammatory cytokines and inhibiting the chemotaxis and adhesion of neutrophils. In addition, Api, as a natural flavonoid (Salehi et al., 2019) extracted from fruits and vegetables, has a wide range of sources and can be obtained easily through the daily diet. It is less expensive than most synthetic drugs and has fewer side effects, which are major advantages for Api as a potential new drug to promote flap survival.

The excision of a large tissue area from the donor is vital for sufficient blood supply in the skin flap and subcutaneous adipose tissue (Wu et al., 2019), which, after transplantation, is initially supplied by nutrients from the donor tissues. Tissue necrosis is the most common complication after skin flap surgery. In contrast to axial pattern flaps, random skin flaps lack axial vascularization and are, therefore, dependent on existing vessels in the surrounding tissues (Cai et al., 2015). However, maintaining a low length-towidth ratio in random skin flaps is crucial for flap survival, as the distal end of the flap may suffer from nutrient deprivation, leading to necrosis (Memarzadeh et al., 2016). Flap survival primarily depends on blood perfusion pressure. In random skin flaps, pedicle muscle vessels are the primary source of blood and nutrients. Therefore, the survival of ultra-long random skin flaps requires constant blood flow at the flap ends, which allows for the formation of new capillaries in the flap (Wang et al., 2011; Ciudad et al., 2017). Although longer operation times can increase flap blood supply and improve flap survival and quality, it can also increase the risk of tissue injury. Therefore, delays in the transplantation process can greatly impact flap survival (Murphy et al., 1985; Haykal et al., 2018; Arslan and Demiroz, 2019; Aydin et al., 2019).

Inflammatory responses also play decisive roles in the survival of random skin flaps. Necrotic areas at the distal flap are characterized by profound coagulative necrosis and inflammatory cell infiltration. 
Additionally, the extent of tissue necrosis has been associated with the degree of inflammatory responses (Perry et al., 2018). Hence, preventing immune responses and inflammation can improve flap survival. The mechanisms underlying tissue necrosis, inflammatory responses, and ischemia reperfusion injury form a complex network that impairs flap survival (Ma et al., 2018). Among these mechanisms, those involved in ischemia reperfusion injury are believed to act upstream in this network. Hypoxic and ischemic flap tissues often exhibit extensive cell membrane damage. During reperfusion, membrane damage and the subsequent rise in the levels of arachidonic acid are further increased (Zachary et al., 1979), promoting neutrophil recruitment and aggravating inflammation. Flap-infiltrating neutrophils produce high levels of ROS, exacerbating tissue damage and inflammation.

Mounting evidence suggests that Api has remarkable antiinflammatory effects. Du $\mathrm{H}$ et al. showed that the Api-rich plant celery strongly suppressed inflammation in rat models, mitigating acute myocardial infarction (Du et al., 2015). Moreover, Li K et al. demonstrated that celery inhibited Toll-like receptor 4 (TLR4) signaling, protecting against acute lung injury after lipopolysaccharide treatment ( $\mathrm{Li}$ et al., 2018). Pang Q et al. showed that celery alleviated ischemia reperfusion injury in the brain by activating the caveolin-1/VEGF axis (Pang et al., 2018)10. In this study, we found that Api treatment reduced the levels of numerous pro-inflammatory cytokines, including TNF$\alpha$, IL-6, and IL-1 $\beta$. This finding suggests that Api may alleviate inflammation by suppressing the production of proinflammatory cytokines and other inflammatory mediators (Charalabopoulos et al., 2019).

Ischemia reperfusion injury is another factor promoting ROS production and oxidative stress and is considered to be the leading cause of skin flap necrosis (Georgopoulos et al., 2012). As an antioxidant, SOD scavenges superoxide anion radicals, thereby maintaining free radical homeostasis during ischemia reperfusion injury (Xu Y. et al., 2017). By contrast, MDA induces membrane damage. Previous studies have shown that Api alleviated cardiac ischemia reperfusion injury (Feng et al., 2018). In this study, we found that Api significantly increased SOD activity and reduced MDA content, suggesting that Api can prevent ischemia reperfusion injury by reducing oxidative stress (Huang et al., 2019).

We also found that Api upregulated VEGF expression. VEGF levels have been associated with vascular regeneration in skin flaps. Early during angiogenesis, VEGF promotes vascular endothelial cell proliferation and differentiation (Gao et al., 2015; Cochran et al., 2016). Previous studies have shown that VEGF reduced skin flap necrosis, prevented flap infection, promoted wound healing, and improved flap survival (Alkharsah 2018; An et al., 2018; Melincovici et al., 2018). In this study, hematoxylin and eosin staining and gelatine/lead oxide angiography results indicated that Api treatment enhanced neovascularization. The density of capillaries in the high-dose Api group was higher than in the low-dose Api group, suggesting a dose-dependent effect. Improved blood flow and nutrient supply can reduce inflammation and improve random skin flap survival.

Compared with other flavonoids, such as quercetin (Mekjaruskul and Sripanidkulchai, 2015; Carullo et al., 2017) and kaempferia brass (Mekjaruskul and Sripanidkulchai, 2015), Api has an improved safety profile. Owing to its antiinflammatory, antioxidant, and pro-angiogenic effects, Api has various applications in medicine and the food industry (Brad and Zhang, 2018). However, low water-solubility and intestinal absorption contribute to the low bioavailability of orally administered Api, limiting its wide application. Moreover, future studies are required to establish the doses of Api that provide maximum clinical benefits.

We conclude that Api improves flap survival, but the underlying mechanism remains to be elucidated fully. Based on existing research, we believe that this mechanism may be related to the TLR4 or caveolin-1/VEGF pathway, which we will consider in future research.

\section{CONCLUSION}

Given its potent antioxidative, angiogenic, and anti-inflammatory capabilities, Api has potential applications for the treatment of ischemic flap tissue in rats. Further studies are necessary to determine the optimal dose and evaluate the safety of Api as a drug, as well as to yield a more detailed understanding of the drug's mechanisms of action. This study was an experimental investigation performed exclusively in rats; whether Api can be applied to the recovery and reconstruction of human skin flap transplantation requires further investigation.

\section{DATA AVAILABILITY STATEMENT}

The original contributions presented in the study are included in the article/Supplementary Material, further inquiries can be directed to the corresponding author.

\section{ETHICS STATEMENT}

The animal study was reviewed and approved by Wenzhou Medical University.

\section{AUTHOR CONTRIBUTIONS}

Conceptualization, XM; Methodology, YL; Software, YL; Validation, XM, WL, and YL; Formal Analysis, JH; Investigation, MF; Resources, MF; Data Curation, YL; Writing-Original Draft Preparation, XM; Writing-Review and Editing, XM; Visualization, DL; Supervision, DL; Project Administration, DL; Funding Acquisition, DL.

\section{FUNDING}

This work was supported by The Science and Technology Innovation Activity Plan of Zhejiang University Students and Xinmiao Talent Plan Project (Grant No: 2020R413019). 


\section{REFERENCES}

Alkharsah, K. R. (2018). VEGF upregulation in viral infections and its possible therapeutic implications. Int. J. Mol. Sci. 19, 1642. doi:10.3390/ijms19061642

An, Y., Liu, W. J., Xue, P., Ma, Y., Zhang, L. Q., Zhu, B., et al. (2018). Autophagy promotes MSC-mediated vascularization in cutaneous wound healing via regulation of VEGF secretion. Cell Death Dis. 9, 58. doi:10.1038/s41419-0170082-8

Arslan, H., and Demiröz, A. (2019). Comparison of subacute and delayed free flap reconstruction in the treatment of open lower extremity fractures. Ulus. Travma Acil Cerrahi Derg. 25, 188-192. doi:10.5505/tjtes.2018.28302

Aydin, O. E., Algan, S., Tan, O., Demirci, E., Keles, O. N., and Kantarci, A. (2019). A novel method for flap delay vacuum assisted flap delay: an experimental study in rabbits. J. Plast. Surg. Hand Surg. 53, 208-215. doi:10.1080/2000656X.2019. 1582425

Bächle, A. C., Mörsdorf, P., Rezaeian, F., Ong, M. F., Harder, Y., and Menger, M. D. (2011). N-acetylcysteine attenuates leukocytic inflammation and microvascular perfusion failure in critically ischemic random pattern flaps. Microvasc. Res. 82, 28-34. doi:10.1016/j.mvr.2011.03.010

Brad, K., and Zhang, Y. (2018). Study on extraction and purification of apigenin and the physical and chemical properties of its complex with lecithin. Pharmacogn. Mag. 14, 203-206. doi:10.4103/pm.pm_159_17

Cai, L., Cao, B., and Lin, D. (2015). Effects of traditional Chinese medicine huangqi injection (radix astragali) on random skin flap survival in rats. J. Reconstr. Microsurg. 31, 565-570. doi:10.1055/s-0035-1555142

Carullo, G., Cappello, A. R., Frattaruolo, L., Badolato, M., Armentano, B., and Aiello, F. (2017). Quercetin and derivatives: useful tools in inflammation and pain management. Future Med. Chem. 9, 79-93. doi:10.4155/fmc-2016-0186

Charalabopoulos, A., Davakis, S., Lambropoulou, M., Papalois, A., Simopoulos, C., and Tsaroucha, A. (2019). Apigenin exerts anti-inflammatory effects in an experimental model of acute pancreatitis by down-regulating TNF- $\alpha$ In Vivo 33, 1133-1141. doi:10.21873/invivo.11583

Ciudad, P., Date, S., Orfaniotis, G., Dower, R., Nicoli, F., Maruccia, M., et al. (2017). Delayed grafting for banked skin graft in lymph node flap transfer. Int. Wound J. 14, 125-129. doi:10.1111/iwj.12570

Cochran, D. B., Gray, L. N., Anderson, K. W., and Dziubla, T. D. (2016). Degradable poly(apigenin) polymer inhibits tumor cell adhesion to vascular endothelial cells. J. Biomed. Mater. Res. B Appl. Biomater. 104, 1438-1447. doi:10.1002/jbm.b.33486

Dolan, R. W., Kerr, D., and Arena, S. (1995). Reducing ischemia-reperfusion injury in rat island groin flaps by dexamethasone and BW755C. Laryngoscope 105, 1322-1325. doi:10.1288/00005537-199512000-00011

Du, H., Hao, J., Liu, F., Lu, J., and Yang, X. (2015). Apigenin attenuates acute myocardial infarction of rats via the inhibitions of matrix metalloprotease- 9 and inflammatory reactions. Int. J. Clin. Exp. Med. 8, 8854-8859.

Ekin, Y., Günüșen, İ., Özdemir, Ö. Y., and Tiftikçioğlu, Y. Ö. (2019). Effect of coagulation status and Co-morbidity on flap success and complications in patients with reconstructed free flap. Turk. J. Anaesthesiol. Reanim. 47, 98-106. doi:10.5152/TJAR.2019.07752

Fang, M., He, J., Ma, X., Li, W., and Lin, D. (2020). Protective effects of dexmedetomidine on the survival of random flaps. Biomed. Pharmacother. 128, 110261. doi:10.1016/j.biopha.2020.110261

Feng, Y., Lu, Y., Liu, D., Zhang, W., Liu, J., Tang, H., et al. (2018). Apigenin-7-O$\beta$-d-(-6" -p-coumaroyl)-glucopyranoside pretreatment attenuates myocardial ischemia/reperfusion injury via activating AMPK signaling. Life Sci. 203, 246-254. doi:10.1016/j.lfs.2018.04.048

Gao, R., Wang, L., Yang, Y., Ni, J., Zhao, L., Dong, S., et al. (2015). Simultaneous determination of oleanolic acid, ursolic acid, quercetin and apigenin in Swertia mussotii Franch by capillary zone electrophoresis with running buffer modifier. Biomed. Chromatogr. 29, 402-409. doi:10.1002/bmc.3290

Georgopoulos, S., Mastorakos, D., Kondi-Pafiti, A., Katsenis, K., Arkadopoulos, N., Kannas, D., et al. (2012). Hydroxyzine, cimetidine and vitamin $\mathrm{C}$ in reducing skin flap necrosis in ischemia-reperfusion injury in rats. A comparative study. J BUON 17, 377-382.

Haykal, S., Roy, M., and Patel, A. (2018). Meta-analysis of timing for microsurgical free-flap reconstruction for lower limb injury: evaluation of the godina principles. J. Reconstr. Microsurg. 34, 277-292. doi:10.1055/s-0037-1621724
He, J. B., Fang, M. J., Ma, X. Y., Li, W. J., and Lin, D. S. (2020). Angiogenic and antiinflammatory properties of azadirachtin A improve random skin flap survival in rats. Exp. Biol. Med. 245, 1672-1682. doi:10.1177/1535370220951896

Hu, J., Li, Z., Xu, L. T., Sun, A. J., Fu, X. Y., Zhang, L., et al. (2015). Protective effect of apigenin on ischemia/reperfusion injury of the isolated rat heart. Cardiovasc. Toxicol. 15, 241-249. doi:10.1007/s12012-014-9290-y

Huang, H., Lai, S., Luo, Y., Wan, Q., Wu, Q., Wan, L., et al. (2019). Nutritional preconditioning of apigenin alleviates myocardial ischemia/reperfusion injury via the mitochondrial pathway mediated by notch1/hes1. Oxid. Med. Cell. Longev. 2019, 7973098. doi:10.1155/2019/7973098

Jiang, R., Lin, C., Jiang, C., Huang, Z., Gao, W., and Lin, D. (2020). Nobiletin enhances the survival of random pattern skin flaps: involvement of enhancing angiogenesis and inhibiting oxidative stress. Int. Immunopharm. 78, 106010. doi:10.1016/j.intimp.2019.106010

Kang, N., Hai, Y., Liang, F., Gao, C. J., and Liu, X. H. (2014). Preconditioned hyperbaric oxygenation protects skin flap grafts in rats against ischemia/ reperfusion injury. Mol. Med. Rep. 9, 2124-2130. doi:10.3892/mmr.2014.2064

Krag, A. E., Eschen, G. T., Damsgaard, T. E., Svaerdborg, M., Steiniche, T., and Kiil, B. J. (2017). Remote ischemic perconditioning attenuates acute inflammation of experimental musculocutaneous flaps following ischemia-reperfusion injury. Microsurgery 37, 148-155. doi:10.1002/micr.30058

Li, K., He, Z., Wang, X., Pineda, M., Chen, R., Liu, H., et al. (2018). Apigenin C-glycosides of Microcos paniculata protects lipopolysaccharide induced apoptosis and inflammation in acute lung injury through TLR4 signaling pathway. Free Radic. Biol. Med. 124, 163-175. doi:10.1016/j.freeradbiomed. 2018.06.009

Lille, S., Boyle, E. M., Jr., Schoeller, T., Suchy, H., and Russell, R. C. (1999). Augmentation of cAMP improves muscle-flap survival and tissue inflammation in response to ischemia/reperfusion injury. J. Reconstr. Microsurg. 15, 37-45. doi:10.1055/s-2007-1000069

Luan, R. L., Meng, X. X., and Jiang, W. (2016). Protective effects of apigenin against paraquat-induced acute lung injury in mice. Inflammation 39, 752-758. doi:10. 1007/s10753-015-0302-2

Ma, J. X., Yang, Q. M., Xia, Y. C., Zhang, W. G., and Nie, F. F. (2018). Effect of 810 $\mathrm{nm}$ near-infrared laser on revascularization of ischemic flaps in rats. Photomed Laser Surg. 36, 290-297. doi:10.1089/pho.2017.4360

Malik, S., Suchal, K., Khan, S. I., Bhatia, J., Kishore, K., Dinda, A. K., et al. (2017). Apigenin ameliorates streptozotocin-induced diabetic nephropathy in rats via MAPK-NF-kappaB-TNF-alpha and TGF-beta1-MAPK-fibronectin pathways. Am. J. Physiol. Ren. Physiol. 313, F414-F422. doi:10.1152/ajprenal.00393.2016

Mekjaruskul, C., and Sripanidkulchai, B. (2015). Pharmacokinetic interaction between Kaempferia parviflora extract and sildenafil in rats. J. Nat. Med. 69, 224-231. doi:10.1007/s11418-014-0882-4

Melincovici, C. S., Boșca, A. B., Şușman, S., Mărginean, M., Mihu, C., Istrate, M. et al. (2018). Vascular endothelial growth factor (VEGF)-key factor in normal and pathological angiogenesis. Rom. J. Morphol. Embryol. 59, 455-467.

Memarzadeh, K., Sheikh, R., Blohmé, J., Torbrand, C., and Malmsjö, M. (2016). Perfusion and oxygenation of random advancement skin flaps depend more on the length and thickness of the flap than on the width to length ratio. Eplasty 16 , e12.

Miean, K. H., and Mohamed, S. (2001). Flavonoid (myricetin, quercetin, kaempferol, luteolin, and apigenin) content of edible tropical plants. J. Agric. Food Chem. 49, 3106-3112. doi:10.1021/jf000892m

Murphy, R. C., Lawrence, W. T., Robson, M. C., and Heggers, J. P. (1985). Surgical delay and arachidonic acid metabolites: evidence for an inflammatory mechanism: an experimental study in rats. Br. J. Plast. Surg. 38, 272-277. doi:10.1016/0007-1226(85)90063-3

Nabavi, S. M., Habtemariam, S., Daglia, M., and Nabavi, S. F. (2015). Apigenin and breast cancers: from chemistry to medicine. Anticancer Agents Med. Chem. 15, 728-735. doi:10.2174/1871520615666150304120643

Pang, Q., Zhao, Y., Chen, X., Zhao, K., Zhai, Q., and Tu, F. (2018). Apigenin protects the brain against ischemia/reperfusion injury via caveolin-1/VEGF in vitro and in vivo. Oxid. Med. Cell. Longev. 2018, 7017204. doi:10.1155/2018/ 7017204

Perry, T. L., Kranker, L. M., Mobley, E. E., Curry, E. E., and Johnson, R. M. (2018). Outcomes in fournier's gangrene using skin and soft tissue sparing flap preservation surgery for wound closure: an alternative approach to wide radical debridement, Wounds 30, 290-299. 
Ren, K., Jiang, T., Zhou, H. F., Liang, Y., and Zhao, G. J. (2018). Apigenin retards atherogenesis by promoting ABCA1-mediated cholesterol efflux and suppressing inflammation. Cell. Physiol. Biochem. 47, 2170-2184. doi:10. $1159 / 000491528$

Salehi, B., Venditti, A., Sharifi-Rad, M., Kręgiel, D., Sharifi-Rad, J., Durazzo, A., et al. (2019). The therapeutic potential of apigenin. Int. J. Mol. Sci. 20, 20. doi:10. 3390/ijms20061305

Skerget, M., Kotnik, P., Hadolin, M., Hras, H. R., Simonic, M., and Knez, Z. (2005). Phenols, proanthocyanidins, flavones and flavonols in some plant materials and their antioxidant activities. Food Chem. 89, 191-198. doi:10.1016/j.foodchem. 2004.02.025

Telange, D. R., Patil, A. T., Pethe, A. M., Fegade, H., Anand, S., and Dave, V. S. (2017). Formulation and characterization of an apigenin-phospholipid phytosome (APLC) for improved solubility, in vivo bioavailability, and antioxidant potential. Eur. J. Pharmaceut. Sci. 108, 36-49. doi:10.1016/j.ejps. 2016.12.009

Wang, J. C., Xia, L., Song, X. B., Wang, C. E., and Wei, F. C. (2011). Transplantation of hypoxia preconditioned bone marrow mesenchymal stem cells improves survival of ultra-long random skin flap. Chin. Med. J. 124, 2507-2511.

Wu, H., Chen, H., Zheng, Z., Li, J., Ding, J., Huang, Z., et al. (2019). Trehalose promotes the survival of random-pattern skin flaps by TFEB mediated autophagy enhancement. Cell Death Dis. 10, 483. doi:10.1038/s41419-0191704-0

Xu, P. F., Fang, M. J., Jin, Y. Z., Wang, L. S., and Lin, D. S. (2017). Effect of oxytocin on the survival of random skin flaps. Oncotarget 3 (8), 92955-92965. doi:10. 18632/oncotarget. 21696
Xu, Y., Zhang, B., Xie, D., Hu, Y., Li, H. L., Zhong, L. L., et al. (2017). Nanoparticlemediated dual delivery of resveratrol and DAP5 ameliorates kidney ischemia/ reperfusion injury by inhibiting cell apoptosis and inflammation. Oncotarget 8 , 39547-39558. doi:10.18632/oncotarget.17135

Zachary, L. S., Robson, M. C., Heggers, J. P., and Kirchner, P. J. (1979). Role of arachidonic acid metabolites in distal dying flap. Surg. Forum 30, 527-528.

Zhang, T., Su, J., Guo, B., Wang, K., Li, X., and Liang, G. (2015). Apigenin protects blood-brain barrier and ameliorates early brain injury by inhibiting TLR4mediated inflammatory pathway in subarachnoid hemorrhage rats. Int. Immunopharm. 28, 79-87. doi:10.1016/j.intimp.2015.05.024

Zhou, Q., Cheng, K. W., Gong, J., Li, E. T. S., and Wang, M. (2019). Apigenin and its methylglyoxal-adduct inhibit advanced glycation end products-induced oxidative stress and inflammation in endothelial cells. Biochem. Pharmacol. 166, 231-241. doi:10.1016/j.bcp.2019.05.027

Conflict of Interest: The authors declare that the research was conducted in the absence of any commercial or financial relationships that could be construed as a potential conflict of interest.

Copyright (C) $2021 \mathrm{Ma}$, Lin, Liu, Li, He, Fang and Lin. This is an open-access article distributed under the terms of the Creative Commons Attribution License (CC BY). The use, distribution or reproduction in other forums is permitted, provided the original author(s) and the copyright owner(s) are credited and that the original publication in this journal is cited, in accordance with accepted academic practice. No use, distribution or reproduction is permitted which does not comply with these terms. 